\title{
Estimativa de parâmetros genéticos da habilidade de permanência aos 48 , 60 e 72 meses de idade em vacas da raça Caracu ${ }^{1}$
}

\section{Sandra Aidar de Queiroz ${ }^{2}$, Gustavo Figueiredo ${ }^{3}$, Josineudson Augusto II de Vasconcelos Silva $^{4}$, Ana Carolina Espasandin ${ }^{5}$, Sarah Laguna Meirelles ${ }^{6}$, João Ademir de Oliveira ${ }^{7}$}

\author{
1 Trabalho de iniciação científica para graduação em Zootecnia - FCAV - UNESP. \\ 2 Departamento de Zootecnia FCAV - UNESP, Bolsista do CNPq. \\ ${ }^{3}$ Zootecnista graduado pela FCAV - UNESP. Bolsista CNPq/PIBIC. \\ ${ }^{4}$ Estação Experimental de Zootecnia de Sertãozinho, IZ/SAA/SP. \\ 5 Departamento de Producción Animal y Pasturas. UDELAR - Uruguay. \\ 6 Doutoranda do Programa de Pós-graduação em Zootecnia - FCAV - UNESP, Jaboticabal - SP. \\ 7 Departamento de Ciências Exatas da FCAV - UNESP - Jaboticabal - SP - CEP: 14884-900.
}

RESUMO - Objetivou-se estimar a herdabilidade da característica habilidade de permanência (HP) em um rebanho de bovinos da raça Caracu visando sua utilização como critério de seleção. A característica em estudo foi definida como a probabilidade de a vaca estar presente no rebanho aos 48 (HP48), aos 60 (HP60) e aos 72 (HP72) meses, desde que possuíssem registros de pelo menos duas lactações nas específicas idades. Observações binárias, com zero $(0=$ fracasso $)$ e um $(1=$ sucesso $)$ foram designadas aos animais. Para análise da HP, foram utilizados dados de 5.487, 4.947 e 4.308 animais aos 48,60 e 72 meses, respectivamente. Os componentes de variância e as herdabilidades foram estimados mediante inferência Bayesiana, via amostragem de Gibbs, pelo programa MTGSAM - threshold, utilizando-se um modelo touro. Foram utilizadas como variáveis explanatórias grupo de contemporâneos, classe de produção de leite na primeira lactação, classe de idade ao primeiro parto e sua interação. As análises forneceram estimativas médias de herdabilidade iguais a 0,28 $\pm 0,07$ para HP48, 0,27 $\pm 0,07$ para HP60 e 0,23 $\pm 0,07$ para HP72. Os resultados evidenciaram que a característica HP apresentou variação aditiva em todas as idades estudadas e, portanto, pode ser empregada como critério de seleção para longevidade produtiva.

Palavras-chave: característica de limiar, dupla-aptidão, longevidade

\section{Estimation of genetic parameters of stayability in Caracu cattle}

\begin{abstract}
The aim of this study was to estimate the heritability of stayability (HP) in Caracu cattle in order to evaluate its usage as a selection criterion for longevity. Stayability was defined as the probability of a cow to be producing in the herd at the ages of 48 (HP48), 60 (HP60) and 72 (HP72) months. Binary observations indicating failure (zero) or success (one) were assigned to records on 5487, 4947 and 4308 cows with at least two lactations at the ages of 48, 60 and 72 months, respectively. Variance components and heritability for HP48, HP60 and HP72 were estimated using a sire model including the fixed effects of contemporary group, classes of milk yield at first calving, classes of age at first calving and the interaction between milk yield and age at first calving classes by Gibbs sampling with the MTGSAM for threshold program. Average heritability estimates for HP48, HP60 and HP72 were $0.28 \pm 0.07,0.27 \pm 0.07$ and $0.23 \pm 0.07$ respectively. These results indicate that HP can be used as selection criterion for longevity in Caracu cattle.
\end{abstract}

Key Words: dual purpose cattle, longevity, threshold trait

\section{Introdução}

O maior tempo de permanência da fêmea em produção no rebanho tem sido associado a maior lucratividade, pois em bovinos o investimento no animal do nascimento até o primeiro parto só começa a ser amortizado na primeira lactação, de modo que seriam necessárias pelo menos duas parições para que os investimentos com a vaca em fase de crescimento fossem recuperados pelo produtor (Ribeiro et al., 2003). Como o tempo de permanência da vaca no rebanho geralmente é determinado pelos desempenhos reprodutivo e produtivo, vacas mais longevas tendem a ser mais rentáveis, pois têm maior oportunidade para retornar a receita sobre custos fixos e variáveis.

Pearson \& Miller (1981) listaram como benefícios do maior tempo de permanência das fêmeas no rebanho a redução do custo de reposição, o aumento no número de fêmeas na faixa etária de maior produção de leite, na qual 
ocorre a desmama de bezerros mais pesados, a redução na quantidade de alimento às novilhas que não estão produzindo e o aumento do descarte voluntário.

Assim, a duração da vida produtiva (DVP) da vaca é uma característica que engloba critérios reprodutivos, produtivos e econômicos e que pode ser utilizada na avaliação da eficiência do animal no sistema de produção. Entretanto, apresenta limitações para ser empregada como critério de seleção, pois, se a medida for feita tardiamente, aumenta o intervalo de gerações e, conseqüentemente, diminui o ganho genético anual.

Uma medida indireta da característica contínua DVPé a habilidade de permanência da vaca no rebanho. A habilidade de permanência (HP) foi definida por Hudson \& Van Vleck (1981) como a probabilidade de a vaca estar presente no rebanho a uma idade específica. Esta é uma característica binária ou categórica, com probabilidade de sucesso (1) caso a vaca permaneça no rebanho, em constante produção, durante determinado período; ou fracasso (0), indicando a não-permanência do animal no período especificado (Silva et al., 2003a). Esta variável pode ser medida em todas as fêmeas em produção no rebanho sem a necessidade de que já tenham encerrado a vida produtiva, como ocorre com a DVP. Como a HP pode ser definida para idades específicas, por exemplo, 48 meses, torna-se mais fácil utilizá-la na avaliação de reprodutores.

Características como a HP, que apresentam apenas duas classes fenotípicas, são denominadas características de limiar (threshold). Segundo Falconer \& Mackay (1996), características de limiar são aquelas que apresentam distribuição descontínua, mas que, quando submetidas à análise genética, são influenciadas por muitos genes, de maneira semelhante à das características quantitativas.

O desenvolvimento de metodologias específicas para análises de dados categóricos veio ao encontro das necessidades dos pesquisadores, pois auxiliou no estudo e viabilizou a utilização de características como habilidade de permanência no rebanho. Como alternativa aos modelos lineares, modelos não-lineares, principalmente o de limiar, têm sido propostos para análises de variáveis categóricas em melhoramento animal. Abdel-Azim \& Berger (1999) destacaram que a verdadeira importância do uso de modelos de limiar seria a estimação de componentes de variância quando se tem apenas um limiar e que a acurácia das estimativas aumentaria se aumentasse o número de categorias da característica se a distribuição estivesse mais próxima da normal ou se houvesse número balanceado de dados.

A raça Caracu é originária do cruzamento nãodirecionado entre os primeiros bovinos trazidos ao Brasil pelos colonizadores; seus mais prováveis ancestrais são animais das raças da península ibérica Transtagana, Minhota, Barrosã, Arouquesa, Mirandesa, Brava e Turino (Carvalho Dias, 1948). Os animais denominados por Caracu Caldeano apresentam pelagem que varia do creme ao vermelho escuro, bom desenvolvimento corporal, chifres grandes e baixos e boa produção de leite. Uma vez que essa raça foi obtida em clima tropical e naturalmente selecionada, o gado Caracu chegou a ser um dos mais criados em todo o País até o início do século $\mathrm{XX}$, no entanto, com a introdução das raças indianas, deixou de ocupar essa posição de destaque e esteve próximo à extinção. Em decorrência da rigorosa seleção natural, os reprodutores Caracu adaptaram-se às condições de monta natural a campo no Brasil e tornaram-se uma das poucas raças com genes de bovinos europeus que suportam servir em monta natural no Brasil Central (Queiroz et al., 2005).

A seleção simultânea para produção de carne e leite e o desenvolvimento de programas de melhoramento que contemplem ambos os objetivos podem ser importantes para o melhoramento de raças de dupla aptidão (Lôbo et al., 2000) e parece ser essencial para preservação da raça Caracu, uma vez que a ausência de direção pode levar à deterioração econômica da atividade e à diminuição sensível do tamanho desta população. Assim, o estudo da HP na raça Caracu contribuiria com mais informações para auxiliar na determinação de objetivos e índices de seleção para este recurso genético singular.

Este trabalho foi realizado com o objetivo de estimar, em rebanhos da raça Caracu, a herdabilidade da característica habilidade de permanência nas idades de 48, 60 e 72 meses.

\section{Material e Métodos}

Trabalhou-se com informações de animais da raça Caracu pertencentes aos rebanhos das Fazendas Chiqueirão e São Francisco, localizadas, respectivamente, nos municípios de Poços de Caldas e Bambuí, em Minas Gerais.

A alimentação dos animais nos meses mais quentes e chuvosos do ano (outubro a maio) foi realizada em regime de pastagens e, nos meses mais frios, quando as geadas eram mais freqüentes e as pastagens pioravam em qualidade, foi fornecida suplementação (silagem de milho) para todos os animais. A alimentação das vacas em lactação foi diferenciada; além do pasto ou da silagem, receberam $2,0 \mathrm{~kg} /$ dia de concentrado no cocho na Fazenda Chiqueirão e 1,0 kg/dia de milho moído com uréia na Fazenda São Francisco.

O manejo reprodutivo baseou-se na utilização de monta natural no período de abril a meados de fevereiro do ano 
seguinte. Utilizou-se um touro para cada retiro com cerca de 50 vacas e lotes de, aproximadamente, 30 novilhas. Até 1995, as novilhas eram acasaladas quando atingiam 24 meses de idade; após 1995, passaram a ser acasaladas aos 17 meses, com $350 \mathrm{~kg}$ de peso vivo, no intuito de melhorar a eficiência reprodutiva.

A ordenha manual, em ambas as fazendas, foi realizada duas vezes ao dia, com o bezerro ao pé, reservando um quarto do úbere (um teto) à alimentação do bezerro. A duração da lactação foi de, aproximadamente, 300 dias $(295,35 \pm 0,34$ dias), período que coincidia com o desmame. Durante o período de aleitamento, os bezerros receberam, a partir de 60 dias de idade, $1,0 \mathrm{~kg} / \mathrm{dia}$ de concentrado no cocho.

O manejo sanitário do rebanho constituiu-se nas vacinações contra febre aftosa, carbúnculo sintomático e brucelose, além do controle de ectoparasitas quando necessário. O combate aos endoparasitas, no entanto, era feito sistematicamente nos meses de maio, agosto e outubro.

Até 1996, os machos eram selecionados aos 10 meses e aos 2 anos de idade e as fêmeas, aos 2 anos de idade, considerando a produção de leite da mãe e o peso individual à desmama. Após 1996, buscou-se diminuir a idade de entrada das fêmeas em reprodução, permitindo-se que todas fossem cobertas. Esta estratégia resultou em aumento do descarte voluntário de vacas em decorrência da baixa produção de leite. Os machos passaram a ser selecionados com base em um índice empírico com ponderação de 50\% para as diferenças esperadas nas progênies (DEP) de produção de leite, $40 \%$ para peso aos 15 meses e $10 \%$ para perímetro escrotal aos 15 meses de idade (Carvalho Dias, 1997). Os animais adultos foram descartados de acordo com a produção leiteira, a fertilidade, a conformação e a idade avançada.

A habilidade de permanência foi definida para as matrizes que permaneceram no rebanho e que apresentaram registros de pelo menos duas lactações até as idades de 48 (HP48), 60 (HP60) e 72 (HP72) meses. Observações binárias, com zero $(0=$ fracasso $)$ e um $(1=$ sucesso $)$ foram designadas aos animais.O sucesso foi atribuído às vacas que alcançaram as idades específicas e o fracasso, àquelas que não as atingiram. Os animais com duas lactações ou mais mas que ainda não haviam atingido a idade específica foram eliminados da avaliação. Assim, a quantidade de animais avaliados diferiu conforme a idade, decrescendo à medida que a idade da avaliação da HP aumentou de 48 para 72 meses de idade. A formação dos arquivos e a análise de consistência dos dados foram realizadas utilizando-se o programa computacional SAS (SAS, 1999).

Análises preliminares foram feitas pelo procedimento LOGISTIC do SAS (SAS, 1999) no intuito de verificar as principais causas de variação na habilidade de permanên- cia. O procedimento LOGISTIC (SAS, 1999) ajusta modelos de regressão linear logístico para dados de resposta binária ou ordinal, pelo método da máxima verossimilhança. A variável-resposta utilizada no modelo foi habilidade de permanência aos 48, 60 e 72 meses, com valores binários de 0 ou 1 . As variáveis explanatórias usadas como efeitos fixos foram: o grupo de contemporâneos; a classe de produção de leite na primeira lactação; a classe de idade da vaca no primeiro parto e a interação dessas classes.

O grupo de contemporâneos (GC) foi criado pela concatenação dos efeitos de rebanho, ano e estação de nascimento da vaca. $\mathrm{O}$ ano de nascimento incluiu o período de 1970 a 1999 para HP48, 1970 a 1997 para HP60 e 1970 a 1995 para HP72, em virtude das especificidades de cada uma das definições de HP utilizadas neste trabalho. As estações consideradas foram primavera (setembro a novembro), verão (dezembro, janeiro e fevereiro), outono (março a maio) e inverno (junho a agosto). Foram estabelecidas as seguintes classes de produção de leite à primeira lactação (PL), em kg: 1) PL < 1.100; 2) $1.100 \leq$ PL<1.400; 3) $1.400 \leq$ $\mathrm{PL}<1.700$; e 4) $\mathrm{PL} \geq 1.700$. A idade ao primeiro parto (IPP), em meses, também foi subdividida em classes, a saber: 1) IPP $\leq 31$; 2) $31<\operatorname{IPP} \leq 34$; 3) $34<$ IPP $\leq 37$; e 4) IPP > 37.

Como demonstrado na Tabela 1 , nos arquivos de HP48, HP60 e HP72 foram analisadas 5.487, 4.947 e 4.308 vacas, filhas de 266, 244 e 211 touros e pertencentes a 141, 131 e 116 grupos de contemporâneos, respectivamente.

Em cada arquivo analisado, foram considerados touros com pelo menos cinco filhas e grupos de contemporâneos (GC) com no mínimo cinco registros. Além disso, foram eliminados GC com médias 0 ou 1 para habilidade de permanência, isto é, sem variabilidade, e animais sem mãe conhecida.

Para a estimação dos componentes de variância e dos parâmetros genéticos da HP nas três idades consideradas, utilizou-se o software MTGSAM for threshold (MultipleTrait Gibbs Sampler for Animal Models, Van Tassel \& Van Vleck, 1995).

Inicialmente, empregou-se um modelo animal para estimação dos componentes de variância. Entretanto, o programa encerrava as iterações apresentando estimativas crescentes da variância aditiva, um fenômeno descrito como blowing-up (Hoeschele et al., 1995). Essa dificuldade tem sido descrita na utilização de modelos de limiar quando os efeitos fixos apresentam muitos níveis (Hoeschele \& Tier, 1995; Moreno et al., 1997) e/ou existe grande variabilidade entre os efeitos. Assim, empregou-se um modelo touro como forma de concentrar as observações das características em menor número de animais e possibilitar a convergência das análises. 
Tabela 1 - Número de observações de habilidade de permanência aos 48 (HP48), aos 60 (HP60) e aos 72 (HP72) meses de idade, de acordo com a classe de produção de leite na primeira lactação ( $\mathrm{PL}, \mathrm{em} \mathrm{kg}$ ), a classe de idade ao primeiro parto (IPP, em meses) e a interação dessas classes (PL x IPP), para vacas da raça Caracu

Table1 - Number of stayabiliy records at the ages of 48 (HP48), 60 (HP60) and 72 (HP72) month by classes of first lactation milk yield (PL, kg), age at first calving (IPP, months) and the interaction between PL and IPP for Caracu cows

\begin{tabular}{lrrr}
\hline PL $(\mathrm{kg})$ & HP48 & HP60 & HP72 \\
\hline $1=$ PL $<1.100$ & 1.546 & 1.313 & 1.047 \\
$2=1.100 \leq \mathrm{PL}<1.400$ & 1.520 & 1.392 & 1.198 \\
$3=1.400 \leq \mathrm{PL}<1.700$ & 1.325 & 1.219 & 1.116 \\
$4=\mathrm{PL} \geq 1.700$ & 1.096 & 1.023 & 947 \\
$\mathrm{IPP}(\mathrm{meses})$ & $\mathrm{HP} 48$ & HP60 & HP72 \\
$1=\mathrm{IPP} \leq 31$ & 1.140 & 765 & 375 \\
$2=31<\mathrm{IPP} \leq 34$ & 1.294 & 1.177 & 987 \\
$3=34<\mathrm{IPP} \leq 37$ & 1.484 & 1.460 & 1.409 \\
$4=\mathrm{IPP}>37$ & 1.569 & 1.545 & 1.537 \\
PL x IPP & HP48 & HP60 & HP72 \\
11 & 438 & 284 & 113 \\
12 & 410 & 359 & 289 \\
13 & 401 & 387 & 370 \\
14 & 297 & 283 & 275 \\
21 & 326 & 235 & 113 \\
22 & 370 & 347 & 296 \\
23 & 431 & 421 & 399 \\
24 & 393 & 389 & 390 \\
31 & 221 & 156 & 97 \\
32 & 330 & 301 & 263 \\
33 & 361 & 357 & 352 \\
34 & 413 & 405 & 404 \\
41 & 155 & 90 & 52 \\
42 & 184 & 170 & 139 \\
43 & 291 & 295 & 288 \\
44 & 466 & 468 & 468 \\
& & & \\
\hline & & &
\end{tabular}

O modelo de limiar utilizado relaciona a resposta observada na escala categórica a uma escala subjacente normal contínua. Assumindo-se que a escala subjacente tem distribuição normal:

$$
\mathrm{U} \theta \sim \mathrm{N}\left(\mathrm{W} \theta, \mathrm{I} \sigma_{\mathrm{e}}^{2}\right)
$$

em que: U é o vetor da escala base de ordem r; $\theta^{\prime}=\left(E^{\prime}, a^{\prime}\right)$, o vetor dos parâmetros de locação de ordem s com EF compondo o conjunto de efeitos fixos (sob o ponto de vista frequentista) e "a" (como efeito aleatório); W, matriz de incidência conhecida de ordem $r$ por s; $I$, matriz identidade de ordem $r$ por $r$; e $\sigma_{\mathrm{e}}{ }^{2}$, a variância residual.

De acordo com o enfoque de inferência Bayesiana, assumiu-se que as distribuições iniciais para os efeitos genéticos e os resíduos seguiram distribuições normais multivariadas:

$\mathrm{P}\left(\mathrm{a} \mid \sigma_{\mathrm{a}}^{2}\right) \sim \mathrm{N}\left(0, \sigma_{\mathrm{a}}^{2}\right)$

$\mathrm{P}\left(\mathrm{e} \mid \sigma_{\mathrm{e}}^{2}\right) \sim \mathrm{N}\left(0, \mathrm{I} \sigma_{\mathrm{e}}^{2}\right)$ em que $\sigma_{\mathrm{a}}{ }^{2}=$ variância genética aditiva. Uma vez que $\sigma_{\mathrm{e}}{ }^{2}$ não é estimável (Gianola \& Foulley, 1983), um valor arbitrário foi-lhe atribuído. Seguindo a sugestão de Silva et al. (2003a), atribuiu-se o valor 1. Foram definidas distribuições flat prior para os efeitos fixos ( $\mathrm{b}^{\prime}=\mathrm{EF}$ ') e para $\sigma_{\mathrm{a}}{ }^{2}$.

Após a definição dos parâmetros do modelo, o encadeamento das duas escalas, categórica e contínua, pode ser estabelecido inequivocamente com a contribuição da probabilidade de uma observação que cai na primeira categoria sendo proporcional a:

$\mathrm{P}(\mathrm{y} V=0 \mid \mathrm{t}, \theta)=\mathrm{P}(\mathrm{UV}<\mathrm{t} \mid \mathrm{t}, \theta)=\Phi\left(\left(\mathrm{t}-\mathrm{w}^{\prime} \mathrm{V} \theta\right) / \sigma_{\mathrm{e}}\right)$

em que: $y \mathbf{V}=$ variável resposta para $a v^{\text {th }}$ observação; $\mathrm{t}=$ valor do limiar para o qual, por não ser estimável, foi fixado um valor arbitrário; UV = valor da variável subjacente para a mencionada observação; $\Phi($ ) = função de distribuição cumulativa de uma variável normal padrão; e $w^{\prime} V=$ vetor coluna de incidência que une $\theta$ à observação $v^{\text {th }}$. Uma vez que as observações são condicionalmente independentes, dado $\theta$, a função de verossimilhança é definida pelo produto das contribuições de cada registro.

De acordo com a inferência Bayesiana, o produto da distribuição anterior pela função de verossimilhança é proporcional à distribuição posterior conjunta de $\theta$ e $\sigma_{\mathrm{a}}{ }^{2}$. Foram então obtidas as distribuições posteriores marginais dos parâmetros usando a amostragem de Gibbs (Gelfand \& Smith, 1990).

O modelo touro utilizado incluiu os efeitos de GC e a variável "classe", composta pela concatenação das variáveis classes de produção de leite na primeira lactação (PL) e classes de idade ao primeiro parto (IPP), definidas anteriormente. A utilização desta variável ("classe") inclui os efeitos principais de PL e IPP e da interação PL $\times$ IPP.

O modelo utilizado pode ser descrito por:

$$
\mathrm{Y}=\mathrm{X} \beta+\mathrm{Ws}+\mathrm{e}
$$

em que: $\mathrm{Y}=$ vetor de observações; $\beta=$ vetor relacionado aos efeitos fixos; $\mathrm{s}=$ vetor relacionado ao efeito genético aditivo de reprodutor; $\mathrm{e}=$ erro aleatório; $\mathrm{X}$ e $\mathrm{W}=$ matrizes de incidência para os respectivos efeitos.

Por se tratar de uma análise unicaracterística, as variâncias estimadas sob modelo touro foram: variância genética aditiva considerada como $4 \sigma_{\mathrm{s}}^{2}$; variância ambiental igual à variância residual, neste caso, fixa com valor 1; variância fenotípica $\left(\sigma_{\mathrm{f}}^{2}\right)$, como a soma de $\left(\sigma_{\mathrm{s}}^{2}+1\right)$ e, conseqüentemente, a herdabilidade estimada sob modelo touro:

$$
h^{2}=\frac{4 \sigma_{s}{ }^{2}}{\left(\sigma_{s}{ }^{2}+1\right)}
$$


em que $\sigma_{\mathrm{s}}^{2}$ é a estimativa do componente de variância genética aditiva atribuído ao touro.

Mediante o software MTGS_THR (Van Tassel et al., 1998), uma cadeia amostral de 700.000 ciclos foi computada, de modo que o período de descarte de dados (burn-in) foi composto dos primeiros 5.000 ciclos. Esse período foi baseado em avaliações subjetivas, por meio de gráficos realizados a partir do programa SAS (SAS, 1996). A freqüência de amostragem (thinning interval ou Skipparameter) foi a cada 30 ciclos e resultou em 23.167 ciclos das amostras das estimativas de distribuição posterior dos componentes de variância. Posteriormente, foram estimados a média, o desvio-padrão, a mediana e o intervalo de confiança das estimativas posteriores da herdabilidade.

Para verificação da convergência das cadeias amostrais, utilizou-se o programa Gibanal, desenvolvido por Van Kaam (1997). Neste programa, a convergência é obtida quando o valor da correlação serial entre os ciclos amostrais é de pequena magnitude (Raftery \& Lewis, 1992).

\section{Resultados e Discussão}

A porcentagem de vacas que permaneceram no rebanho diminuiu à medida que a idade considerada aumentou (Figura 1), evidenciando as dificuldades encontradas pelo produtor na manutenção de vacas com bom desempenho produtivo e reprodutivo com o passar do tempo. Aos 48 meses, grande parte das vacas incluídas no rebanho continuou em produção, enquanto, aos 72 meses de idade, mais de $50 \%$ delas já haviam sido descartadas.

O efeito de GC foi significativamente importante $(\mathrm{P}<0,001)$ para a HP aos 48, 60 e 72 meses (Tabela 2). Os efeitos considerados no GC estão relacionados ao manejo das empresas, à disponibilidade e à qualidade do alimento, além das variações ambientais e de manejo. A variável GC deve, portanto, ser incluída no modelo para estimação de parâmetros genéticos.

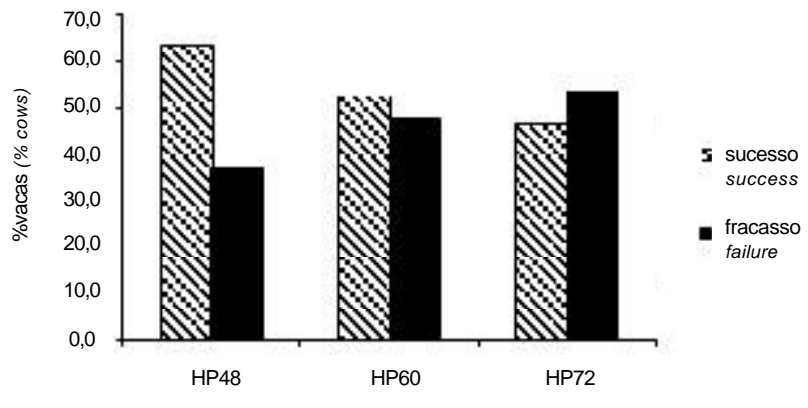

Figura 1 - Porcentagem de sucesso e fracasso em vacas da raça Caracu para a habilidade de permanência aos 48 meses (HP48), aos 60 meses (HP60) e aos 72 meses (HP72) de idade.

Figure 1 - Percentage of success or failure of stayability at the ages of 48 (HP48), 60 (HP60) and 72 months (HP72) in Caracu cattle.

Observou-se que o efeito da classe de produção de leite à primeira lactação foi importante fonte de variação para a permanência da vaca no rebanho nas três idades estudadas. No entanto, as diferentes classes de idade da vaca ao primeiro parto só foram importantes $(\mathrm{P}<0,001)$ para HP48. A interação PL $\times$ IPP, por sua vez, teve efeito significativo $(\mathrm{P}<0,001)$ sobre a habilidade de permanência às diferentes idades, evidenciando comportamento distinto dessas duas variáveis quando consideradas em conjunto (Tabela 2).

A interação significativa PL $\times$ IPP evidencia a importância de considerar os efeitos conjuntos destas variáveis na análise genética da HP(Tabela 3). Esses resultados refletiram a eficiência dos critérios de seleção adotados no rebanho, no qual a produção de leite das mães na primeira lactação era usada para seleção de machos e o desempenho leiteiro na primeira lactação era critério para descarte voluntário de fêmeas. Além disso, o manejo do rebanho até 1996 não permitia que novilhas fossem acasaladas antes dos 24 meses de idade. A partir de então, as novilhas passaram a ser submetidas à monta por volta dos 17 meses de idade.

Tabela 2 - Resumo da análise de regressão logística utilizando-se os efeitos de grupo de contemporâneos (GC), produção de leite na primeira lactação $(P L)$, idade ao primeiro parto (IPP) e interação $P L \times I P P$ sobre a habilidade de permanência no rebanho aos 48 meses (HP48), aos 60 meses (HP60) e aos 72 meses (HP72) em vacas da raça Caracu

Table 2 - Summary of the logistic regression analysis for the model including the effects of contemporary group, first lactation milk yield (PL), age at first calving (IPP and PIXIPP interaction of stayability at the ages of 48 [HP48], 60 [HP60] and 72 [HP72]) months in Caracu cattle

\begin{tabular}{|c|c|c|c|c|c|c|}
\hline \multirow[b]{2}{*}{ Efeito (Effect) } & \multicolumn{2}{|c|}{ HP48 } & \multicolumn{2}{|c|}{ HР60 } & \multicolumn{2}{|c|}{ HP72 } \\
\hline & $\mathrm{GL}^{1}$ & $\chi^{2}$ & GL & $\chi^{2}$ & GL & $\chi^{2}$ \\
\hline Intercepto (Intercept) & 1 & $467,69 *$ & 1 & $32,10 *$ & 1 & $12,44 *$ \\
\hline GC & 140 & $613,17 *$ & 130 & $415,96 *$ & 115 & $246,98 *$ \\
\hline PL & 3 & $762,38 *$ & 3 & $772,84 *$ & 3 & $528,59 *$ \\
\hline IPP & 3 & $55,28 *$ & 3 & 5,45 & 3 & 2,52 \\
\hline PL x IPP & 9 & $44,61 *$ & 9 & $42,49 *$ & 9 & $25,69 *$ \\
\hline
\end{tabular}

${ }^{1} \mathrm{GL}=$ graus de liberdade; * $\mathrm{P}<0,001 ; \chi^{2}=$ qui-quadrado.

${ }^{1} \mathrm{GL}=$ degrees of freedom; $\chi^{2}=$ chi- square. 
Tabela 3 - Número de observações de habilidade de permanência no rebanho aos 48 (HP48), aos 60 (HP60) e aos 72 (HP72) meses de idade considerando a interação entre a classe de produção de leite na primeira lactação $(\mathrm{PL}, \mathrm{em} \mathrm{kg})$ e a classe de idade ao primeiro parto (IPP, em meses)

Table 3 - Number of records of stayability at the ages of 48 (HP48), 60 (HP60) and 72 (HP72) months for the interaction effect between classes of first lactation milk yield (PL, $\mathrm{kg})$ and age at first calving (IPP, months) in Caracu cattle

\begin{tabular}{|c|c|c|c|c|c|c|}
\hline \multirow[b]{2}{*}{$\mathrm{PL} \times \mathrm{IPP}$} & \multicolumn{2}{|c|}{ HP48 } & \multicolumn{2}{|c|}{ HP60 } & \multicolumn{2}{|c|}{ HP72 } \\
\hline & $\begin{array}{c}\text { Fracasso } \\
\text { Failure }\end{array}$ & $\begin{array}{l}\text { Sucesso } \\
\text { Success }\end{array}$ & $\begin{array}{c}\text { Fracasso } \\
\text { Failure }\end{array}$ & $\begin{array}{l}\text { Sucesso } \\
\text { Success }\end{array}$ & $\begin{array}{c}\text { Fracasso } \\
\text { Failure }\end{array}$ & $\begin{array}{r}\text { Sucesso } \\
\text { Success }\end{array}$ \\
\hline 11 & 317 & 121 & 240 & 44 & 100 & 13 \\
\hline 12 & 289 & 121 & 288 & 71 & 260 & 29 \\
\hline 13 & 238 & 163 & 296 & 91 & 306 & 64 \\
\hline 14 & 161 & 136 & 211 & 72 & 229 & 46 \\
\hline 21 & 190 & 136 & 138 & 97 & 69 & 44 \\
\hline 22 & 175 & 195 & 199 & 148 & 186 & 110 \\
\hline 23 & 141 & 290 & 246 & 175 & 259 & 140 \\
\hline 24 & 98 & 295 & 203 & 186 & 254 & 136 \\
\hline 31 & 102 & 119 & 73 & 83 & 45 & 52 \\
\hline 32 & 93 & 237 & 109 & 192 & 109 & 154 \\
\hline 33 & 58 & 303 & 108 & 249 & 147 & 205 \\
\hline 34 & 30 & 383 & 97 & 308 & 156 & 248 \\
\hline 41 & 65 & 90 & 36 & 54 & 20 & 32 \\
\hline 42 & 40 & 144 & 44 & 126 & 40 & 99 \\
\hline 43 & 21 & 270 & 33 & 262 & 45 & 243 \\
\hline 44 & 18 & 448 & 42 & 426 & 85 & 383 \\
\hline Total & 2.036 & 3.451 & 2.363 & 2.584 & 2.310 & 1.998 \\
\hline
\end{tabular}

$1=\mathrm{PL}<1.100 \mathrm{~kg} ; 2=1.100 \leq \mathrm{PL}<1.400 \mathrm{~kg} ; 3=1.400 \leq \mathrm{PL}<1.700 \mathrm{~kg} ; 4=\mathrm{PL} \geq 1.700 \mathrm{~kg}$.

$1=\mathrm{IPP} \leq 31$ meses (months); $2=31<\mathrm{IPP} \leq 34$ meses (months), $3=34<\mathrm{IPP} \leq 37$ meses (months); $4=\mathrm{IPP}>37$ meses (months).

Verifica-sena Tabela 3 a ocorrência de diferente distribuição das informações de sucesso e fracasso nos arquivos de HP relativos às três idades, principalmente, nas classes de menor PL (1) e de menor IPP (1). Menor número de sucessos foi atribuído às vacas destas categorias nos arquivos de HP60 e HP72 em comparação ao de HP48. Houve melhor homogeneidade na distribuição das classes de IPP no arquivo de HP48 quando comparado aos de HP60 e HP72 (Tabela 1), pois este arquivo contava com maior número de vacas acasaladas mais precocemente (fêmeas nascidas entre 1970 e 1999). Além disso, verificaram-se (Tabela 3) maiores freqüências de sucessos para vacas com maior PL e mais velhas (classes 33, 34, 43 e 44 para interação PL $\times$ IPP) para as três características (HP48, HP 60 e HP72). Os resultados obtidos neste estudo estão de acordo com dados da literatura, que reforçam a importância do desempenho na primeira lactação e da idade ao primeiro parto sobre a permanência de animais no rebanho. Carvalho Dias (1997), estudando vacas Caracu, estimou correlação genética de 0,91 entre produção de leite na primeira lactação (PL1) e longevidade e de e 0,90 entre PL1 e vida produtiva, evidenciando a forte associação entre estas características. Valencia et al. (2001) recomendaram a utilização da PL1 como critério de seleção a fim de obter maior longevidade. Bertazzo et al. (2004) demonstraram, mediante a correlação genética entre longevidade e IPP, que novilhas mais precoces tenderam a permanecer por menos tempo no rebanho. Queiroz \& Pelicioni (2001) estimaram, para a raça Caracu, correlação genética entre IPP e duração da vida produtiva igual a-0,09, valor não diferente de zero $(\mathrm{P}>0,01)$.

Como demonstrado na Tabela 4, ao utilizar o método gráfico do SAS (SAS, 1999) ou o programa Gibanal (Van Kaam, 1997), verificaram-se valores bem próximos para a média e a mediana posteriores da variância genética de touro, o que indica distribuição normal quase perfeita. No método gráfico, houve uma abordagem um pouco mais conservadora, pois o descarte amostral (burn in) para se iniciar a convergência dos dados foi maior. O tamanho da cadeia também foi maior, o que gerou maior número de amostras, implicando maior confiabilidade dos resultados observados. Essa abordagem pode ser realizada quando não há limitações de tempo e/ou computacional para a realização das análises.

As médias dos valores de herdabilidade obtidas para a HP nas três idades foram semelhantes (a da HP72 foi um pouco menor $0,2311 \pm 0,0693)$, indicando a presença de variação genética aditiva para as características estudadas. Os valores estimados para as medianas foram muito próximos dos descritos para as médias, o que evidencia simetria de valores, um indicativo da convergência das análises e da coerência das amostras (Tabela 5). As estimativas obtidas foram moderadas e sugerem a possibilidade de obtenção de resposta à seleção se a habilidade de permanência for empregada como critério de seleção. O uso de touros 
Tabela 4 - Estimativa das distribuições posteriores da variância genética aditiva de touro $\left(\sigma_{\mathrm{s}}{ }^{2}\right)$, obtidas pelo método gráfico e pelo programa Gibanal, para a habilidade de permanência no rebanho aos 48 (HP48), aos 60 (HP60) e aos 72 (HP72) meses de idade

Table 4 - Estimates of posterior distribution of sire additive genetic variance $\left(\sigma_{s}^{2}\right)$ of stayability at the ages of 48 (HP48), 60 (HP60) and 72 (HP72) months, using the graphic method and the Gibanal software

\begin{tabular}{|c|c|c|c|c|c|c|}
\hline \multirow[b]{2}{*}{$\sigma_{\mathrm{s}}^{2}$} & \multicolumn{2}{|c|}{ HP48 } & \multicolumn{2}{|c|}{ HP60 } & \multicolumn{2}{|c|}{ HP72 } \\
\hline & Gráfico $^{1}$ & Gibanal & Gráfico & Gibanal & Gráfico & Gibanal \\
\hline $\begin{array}{l}\text { Cadeia amostral } \\
\text { Descarte inicial } \\
\end{array}$ & $\begin{array}{c}700.000 \\
5.000\end{array}$ & $\begin{array}{c}23.167 \\
74\end{array}$ & $\begin{array}{c}700.000 \\
5.000\end{array}$ & $\begin{array}{c}23.167 \\
3\end{array}$ & $\begin{array}{c}700.000 \\
5.000\end{array}$ & $\begin{array}{c}23.167 \\
44\end{array}$ \\
\hline Freqüência de amostragem ${ }^{4}$ & 30 & 158 & 30 & 51 & 30 & 77 \\
\hline Número de amostras ${ }^{5}$ & 23.167 & 147 & 23.167 & 455 & 23.167 & 301 \\
\hline Média $^{6}$ & 0,0765 & 0,0751 & 0,0732 & 0,0747 & 0,0626 & 0,0640 \\
\hline Desvio-padrão $^{7}$ & 0,0204 & 0,0200 & 0,0206 & 0,0220 & 0,0196 & 0,0210 \\
\hline Mediana $^{8}$ & 0,0747 & 0,0723 & 0,0712 & 0,0720 & 0,0610 & 0,0620 \\
\hline $\begin{array}{l}\text { Região de credibilidade } \\
\text { a } 95 \% 9\end{array}$ & 0,0415 a 0,1208 & 0,0410 a 0,1212 & 0,0386 a 0,1183 & 0,0394 a 0,1284 & 0,0291 a 0,1061 & 0,0294 a 0,1118 \\
\hline
\end{tabular}

Tabela 5 - Estatísticas descritivas das estimativas de herdabilidade da característica habilidade de permanência no rebanho aos 48 (HP48), aos 60 (HP60) e aos 72 (HP72) meses de idade

Table 5 - Descriptive statistics for heritability estimates of stayability at the ages of 48 (HP48), 60 (HP60) and 72 (HP72) months in Caracu cattle

\begin{tabular}{lccrr}
\hline \multicolumn{4}{c}{$\begin{array}{c}\text { Herdabilidade } \\
\text { Heritability }\end{array}$} \\
\cline { 2 - 5 } & ${\text { Média } \pm \mathrm{DP}^{1}}$ Mediana $^{2}$ & Mínimo $^{3}$ & Máximo $^{4}$ \\
\hline HP48 & $0,2773 \pm 0,0665$ & 0,2728 & 0,0723 & 0,5724 \\
HP60 & $0,2710 \pm 0,0695$ & 0,2655 & 0,0529 & 0,6162 \\
HP72 & $0,2311 \pm 0,0693$ & 0,2264 & 0,0408 & 0,5981 \\
\hline
\end{tabular}

$\mathrm{DP}$ = desvio-padrão da estimativa de herdabilidade (standard deviation of heritability estimate).

${ }^{1}$ Mean \pm standard deviation; ${ }^{2}$ Median; ${ }^{3}$ Minimum: ${ }^{4}$ Maximum

geneticamente superiores para essa característica deve resultar em filhas com maior probabilidade de permanecerem produtivas no rebanho até 72 meses de idade, trazendo benefícios econômicos duradouros, com efeitos a médio e a longo prazo, uma vez que os ganhos obtidos com o uso contínuo de reprodutores geneticamente melhorados são permanentes e cumulativos.

As estimativas de herdabilidade obtidas por Silva et al. (2003a,b) foram semelhantes (0,21 e 0,22, respectivamente) às encontradas neste estudo para HP72, também utilizando modelo de limiar. Por outro lado, Teixeira et al. (2003) observaram, na raça Holandesa, valores iguais a 0,01 para HP48, 0,02 para HP60 e 0,05 para HP72, enquanto Valencia et al. (2001) obtiveram 0,05 para a herdabilidade da HP48 na mesma raça, no México.

Outro aspecto a ser analisado é que, ao utilizar a HP como critério de seleção, outras características também poderiam ser melhoradas mediante seleção indireta, por exemplo, a produção de leite e a assiduidade reprodutiva. $\mathrm{Na}$ raça Caracu, Queiroz et al. (2005) estimaram valor econômico de $\mathrm{R} \$ 10.016,67$ para a duração da vida produtiva das vacas em anos (DVP). Esse valor monetário representa o acréscimo no lucro anual do rebanho para cada unidade de melhoramento obtida na característica, evidenciando a grande importância econômica da permanência das vacas no rebanho. Entretanto, a herdabilidade da DVP estimada por Queiroz \& Pelicioni (2001) para a raça Caracu foi de apenas 0,14 , quase metade do valor obtido neste estudo para a HP nas diferentes idades. Assim, como HP e DVP podem ser entendidas como diferentes expressões da mesma característica, o emprego da HP na seleção do rebanho Caracu poderá ter impacto econômico consideravelmente maior que o da DVP.

\section{Conclusões}

Na raça Caracu, a habilidade de permanência no rebanho às idades de 48, 60 e 72 meses apresentou variação genética aditiva e, portanto, pode responder à seleção.

\section{Agradecimento}

Ao Zootecnista MSc. André Stein Carvalho Dias, pela cessão dos dados utilizados nesta pesquisa e pelos esclarecimentos sobre manejo e alimentação dos animais.

\section{Literatura Citada}

ABDEL-AZIM, G.A.; BERGER, P.J. Properties of threshold model predictions. Journal of Animal Science, v.77, p.582-590, 1999.

BERTAZZO, R.P.; FREITAS, R.T.F.; GONÇALVES, T.M. et al. Parâmetros genéticos de longevidade e produtividade de fêmeas da raça Nelore. Revista Brasileira de Zootecnia, v.33, n.5, p.1118-1127, 2004. 
CARVALHO DIAS, E. O gado Caracu sob o sistema de retiros. Revista Ceres, v.7, n.22, p.338-402, 1948.

CARVALHO DIAS, A.S. Estudo das características produtivas e de permanência em um rebanho de bovinos da raça Caracu. Jaboticabal: Universidade Estadual Paulista, 1997. 65p. Dissertação (Mestrado em Zootecnia) - Universidade Estadual Paulista, 1997.

FALCONER, D.S.; MACKAY, T.F.C. Threshold characters. In: FALCONER, D.S.; MACKAY, T.F.C. (Eds.) Introduction to quantitative genetics. 4.ed. Essex: Longman, 1996. p.299-311.

GELFAND, A.E.; SMITH, A.F.M. Sampling based approaches to calculating marginal densities. Journal of American Statistical Association, v.85, p.398-409, 1990.

GIANOLA, D.; FOULLEY, J.L. Sire evaluation for ordered categorical data with a threshold model. Genetics, Selection and Evolution, v.15, p.201-224, 1983.

HOESCHELE, I.; TIER, B.; GRASER, H.U. Multiple-trait genetic evaluation for one polycothomous trait and several continuous traits with missing data and unequal models. Journal of Animal Science, v.73, p.1609-1627, 1995.

HOESCHELE, I.; TIER, B. Estimation of variance components of threshold characters by marginal posterior modes and means via Gibbs sampling. Genetics, Selection and Evolution, v.27, n.6, p.519-540, 1995.

HUDSON, G.E.S.; van VLECK, L.D. Relations between production and stayability in Holstein cattle. Journal of Dairy Science, v.64, p.2246-2250, 1981.

LÔBO, R.N.B.; MADALENA, F.E.; PENNA, V.M. Avaliação de um esquema de seleção para bovinos Zebus de dupla aptidão. Revista Brasileira de Zootecnia, v.29, n.5, p.1349-1360, 2000.

MORENO, C.; SORENSEN, D.; GARCIA CORTES, L.A. et al. On biased inference about variance components in the binary threshold model. Genetics, Selection and Evolution, v.29, n.2, p.145-160, 1997.

PEARSON, R.E.; MILLER, R.H. Economic definition of total performance, breeding goals and breeding values for dairy cattle. Journal of Dairy Science, v.64, p.857-869, 1981.

QUEIROZ, S.A.; PELICIONI, L.C. Estimativas dos parâmetros genéticos da vida produtiva de vacas da raça Caracu. In: REUNIÓN DE LA ASOCIACIÓN LATINOAMERICANA DE PRODUCCIÓN ANIMAL, 17., 2001, Havana. Resumenes... Havana: Asociación Latinoamericana de Producción Animal/ Softcal [2001]. (CD-ROM).

QUEIROZ, S.A.; PELICIONI, L.C.; SILVA, B.F. et al. Índices de seleção para um rebanho Caracu de duplo propósito. Revista Brasileira de Zootecnia, v.32, n.6, p. 1737-1746, 2005 (supl. 1).

RAFTERY, A.E.; LEWIS, S.M. How many iterations in the Gibbs sampler? In: BERNARDO, J.M.; BERGER, J.O.; DAWID, A.P.;
SMITH, A.F.M. (Eds.) Bayesian statistics. 4.ed. Oxford: Oxford University Press, 1992. p.765-776.

RIBEIRO, A.C.; MCALLISTER, A.J.; QUEIROZ, S.A. Efeito das taxas de descarte sobre medidas de rentabilidade de vacas leiteiras. Revista Brasileira de Zootecnia, v.34, n.3, p.827-837, 2005

STATISTICAL ANALYSIS SYSTEM - SAS. SAS/STAT language guide. version 6. 3.ed. Cary: 1999. 530p.

SILVA, J.A. II V.; ELER, J.P.; FERRAZ, J.B.S. et al. Análise genética da habilidade de permanência em fêmeas da raça Nelore. Revista Brasileira de Zootecnia, v.32, n.3, p.598-604, 2003a.

SILVA, J.A.II V.; ELER, J.P.; FERRAZ, J.B.S. et al. Relação genética entre habilidade de permanência e perímetro escrotal em animais da raça Nelore. In: REUNIÃO ANUAL DA SOCIEDADE BRASILEIRA DE ZOOTECNIA, 40., 2003, Santa Maria. Anais... Santa Maria: Sociedade Brasileira de Zootecnia/Infovia, [2003b]. (CD-ROM).

SNELLING, W.M.; GOLDEN, B.L.; BOURDON, R.M. Within-herd genetic analyses of stayability of beef cattle. Journal of Animal Science, v.73, p.993-1001, 1995.

TEIXEIRA, N.M.; FERREIRA, W.J.; TORRES, R.A. et al. Parâmetros genéticos para características de longevidade de vacas da raça Holandesa no Estado de Minas Gerais. In: REUNIÃO ANUAL DA SOCIEDADE BRASILEIRA DE ZOOTECNIA, 40., 2003, Santa Maria. Anais... Santa Maria: Sociedade Brasileira de Zootecnia/Infovia, [2003]. (CD-ROM).

VALENCIA, P.M.; RUIZ, L.F; MONTALDO, V.H. et al. Parametros geneticos en caracteristicas de longevidad y produccion de leche en ganado Holstein en Mexico. In: REUNIÓN DE LA ASOCIACIÓN LATINOAMERICANA DE PRODUCCIÓN ANIMAL, 17., 2001, Havana. Resumenes... Havana: Asociación Latinoamericana de Producción Animal/Softcal [2001]. (CD-ROM).

Van KAAM, J.B.C.H.M. GIBANAL: analyzing program for Markov Chain Monte Carlo sequences. version 2.3, 1997. (CD-ROM).

Van TASSELL, C.P.; Van VLECK, L.D. A manual for use the MTGSAM. A set of FORTRAN programs to apply Gibbs sampling to animal models for variance component estimation (DRAFT). Lincoln: U.S. Departament of Agriculture, Agricultural Research Service. 1995. 86p.

Van TASSELL, C.P.; Van VLECK, L.D.; GREGORY, K.E. Bayesian analysis of twinning and ovulation rates using a multiple-trait threshold model and Gibbs sampling. Journal of Animal Science, v.76, p.2048-2061, 1998. 